\title{
Complete RF Analysis of Compound FETs Based on Transient Monte Carlo Simulation
}

\author{
S. BABIKER, A. ASENOV*, N. CAMERON, S. P. BEAUMONT and J. R. BARKER \\ Department of Electronics and Electrical Engineering, Nanoelectronics Research Centre, Glasgow University, \\ Glasgow G12 8LT, Scotland, UK
}

\begin{abstract}
In this paper we described a complete methodology to extract the RF performance of 'real' compound FETs from time domain Ensemble Monte-Carlo (EMC) simulations which can be used for practical device design. The methodology is based on transient finite element EMC simulation of realistic device geometry. The extraction of the terminal current is based on the Ramo-Shockley theorem. Parasitic elements like the gate and contact resistances are included in the RF analysis at the post-processing stage. Example of the RF analysis of pseudomorphic HEMTs illustrates our approach.
\end{abstract}

Keywords: Monte-Carlo, RF analysis, compound FETs, simulation

\section{INTRODUCTION}

The remarkable RF performance of compound FETs such as GaAs MESFETs and InGaAs HEMTs with channel lengths down to $0.1 \mu \mathrm{m}$ is due to well pronounced velocity overshoot. The use of simulation for predictive analysis and design of such devices require in many cases the employment of full scale EMC technique [1-3]. However, most of the published EMC studies of compound FETs consider simplified device geometry and focus mainly on the transport physics and the effect of the enhanced channel velocity on the DC device characteristics. Far more important for the proper design of modern short channel compound FETs is the RF performance which is determined not only by the high field transport but also by the device geometry and the surface effects. The T- or $\Gamma$-shaped gate, the gate recess and the passivation in such devices critically affect the device parasitics and the overall RF device performance.

In this paper we describe a methodology based on the EMC simulation to investigate the RF performance of FETs. The terminal currents are estimated using the Ramo-Shockley theorem. The device parasitics are included through the proper finite-element description of the gate and recess shapes. The external parasitics are included in the post-processing stage.

\footnotetext{
* Corresponding author.
} 


\section{TRANSIENT CURRENT}

The Heterojunction 2D Finite Element simulator $(\mathrm{H} 2 \mathrm{~F})$ and its Monte-Carlo module are described in detail elsewhere $[4,5]$. In the MC simulation the total transient terminal current in response to a step change in the applied voltages required for the $y$-parameter extraction is the sum of the particle current and the displacement current. According to the Ramo-Shockley theorem [6] the instantaneous transient current in electrode $i$ due to $N$ discrete moving charges within the device is given by the sum of the current $I_{i}^{\prime}(t)$ solely contributed by the movement of the $N$ charged particles with fixed potentials at electrodes and the current $I_{i}^{\prime \prime}(t)$ induced due to the time-varying potentials of the electrodes through the capacitive coupling through the electrodes. The current $I_{i}^{\prime}(t)$ is given by

$$
I_{i}^{\prime}(t)=-\sum_{j=1}^{N} q_{j} v_{j}(t) \cdot \nabla f_{i}
$$

where $q_{j}$ is the charge of the super-particle, $v_{j}(t)$ is the velocity of the particle and $f_{i}$ is the solution of the Laplace equation $\nabla \cdot\left(\varepsilon \nabla f_{i}\right)=0$ with a unit voltage applied to electrode $i$, while all other electrodes are grounded.

The current $I_{i}^{\prime \prime}(t)$ associated with the time varying potential is calculated from the capacitance matrix components $C_{i j}$ associated with the electrodes of the simulated device. The capacitance matrix components are obtained from the solution of the Laplace equation as $C_{i j}=\Delta Q_{i} / \Delta V_{j}$ where $\Delta Q_{i}$ is the change in the electrode charge in response to a change in the potential of a particular electrode. When a step perturbation $\Delta V_{j}$ of the terminal voltage is applied $I_{i}^{\prime \prime}(t)$ flows only during the first time step $\Delta t$ and is given by:

$$
I_{i}^{\prime \prime}(t)=C_{i j} \frac{\Delta V_{j}}{\Delta t}
$$

The displacement current during the remaining part of the transient is related to the charges induced on the electrode by the moving particles in the device associated with the redistribution of the mobile charge and is accounted for by Eq. (1).

\section{RF ANALYSIS}

The flow diagram of the complete time domain RF EMC analysis is given in Figure 1. The $y$-parameters are calculated by Fourier decomposition of the current transients obtained in response to step perturbations in the terminal voltages [7]. The cutoff frequency of the simulated device $f_{T}^{\text {sim }}$ is extracted by solving $\log \left[G_{c}^{\operatorname{sim}}(\log f)\right]=0$ where $G_{c}=d I_{d} / d I_{g}$ is the current gain expressed as a function of $y$-parameters.

In order to extract the maximum frequency of oscillation of the simulated device $f_{\max }^{\text {sim }}$ the $y$-parameters are transformed into $s$-parameters $S^{\mathrm{sim}}$. The maximum frequency of oscillation $f_{\max }^{\mathrm{sim}}$

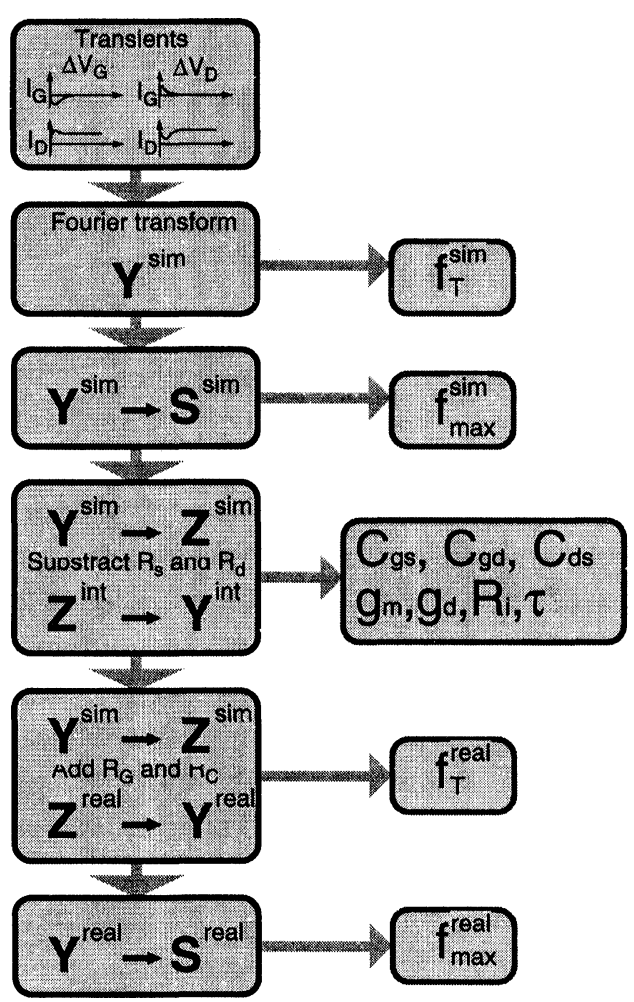

FIGURE 1 Flow diagram of the RF EMC analysis. 
is then extracted by solving $\log \left[M A G^{\operatorname{sim}}(\log f)\right]=0$ where $M A G^{\text {sim }}$ is the maximum available gain.

For typical MESFET and HEMT simulation domains, we have adopted the equivalent circuit model presented in Figure 2. The gate resistance, the contact resistances and any external inductive components are excluded from the EMC simulation. The source $R_{s 1}$ and the drain $R_{d 1}$ resistances in Figure 2 represent the resistance of the regions between the gate and the source and drain ohmic contacts respectively. To extract accurately the small signal equivalent circuit the $y$-parameters of the simulated device $\mathbf{Y}^{\text {sim }}$ are transformed into $z$ parameters $\mathbf{Z}^{\text {sim }}$. The estimated source and drain resistances $R_{s 1}$ and $R_{d 1}$ are subtracted from $\mathbf{Z}^{\text {sim }}$ to obtain the $z$-parameters $\mathbf{Z}^{\text {int }}$ of the 'intrinsic' device. Finally $\mathbf{Z}^{\text {int }}$ are transformed back into $y$ parameters $\mathbf{Y}^{\text {int }}$ from which the components of the 'intrinsic' small signal circuit can be analytically extracted [7]:

In order to evaluate the cut-off frequency $f_{T}^{\text {real }}$ and the maximum frequency of oscillations $f_{\max }^{\text {real }}$ of the 'real' device the gate resistance $R_{g}$, the contact resistances $R_{c}$ and eventually the inductive components $L_{g}, L_{s}$ and $L_{d}$ first have to be incorporated in the $z$-parameters of the real device $\mathbf{Z}^{\text {real }}$ which are then transformed into $\mathbf{Y}^{\text {real }}$. From $\mathbf{Y}^{\text {real }}$ and $S^{\text {real }}$ the figures of merit of the 'real' device we can estimate $f_{T}^{\text {real }}$ and $f_{\mathrm{max}}^{\text {real }}$. The intrinsic minimum noise figure is also evaluated from the two-port $y$ parameters and the current traces.

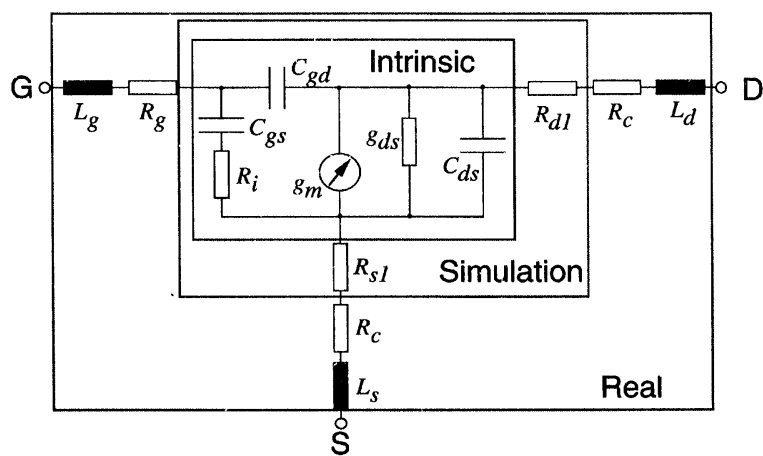

FIGURE 2 Small signal equivalent circuit of a real FET. Figure also shows the 'intrinsic' and 'simulated' device equivalent circuit.

\section{RESULTS}

We apply the described RF analysis in the simulation of a $120 \mathrm{~nm}$ T-gate InGaAs channel pHEMT with $22 \mathrm{~nm}$ gate to channel separation [8] The $y$-parameters extracted from the Fourier decomposition of the transients are shown in Figures $3 \mathrm{a}, \mathrm{b}$. Table I summarises the small signal
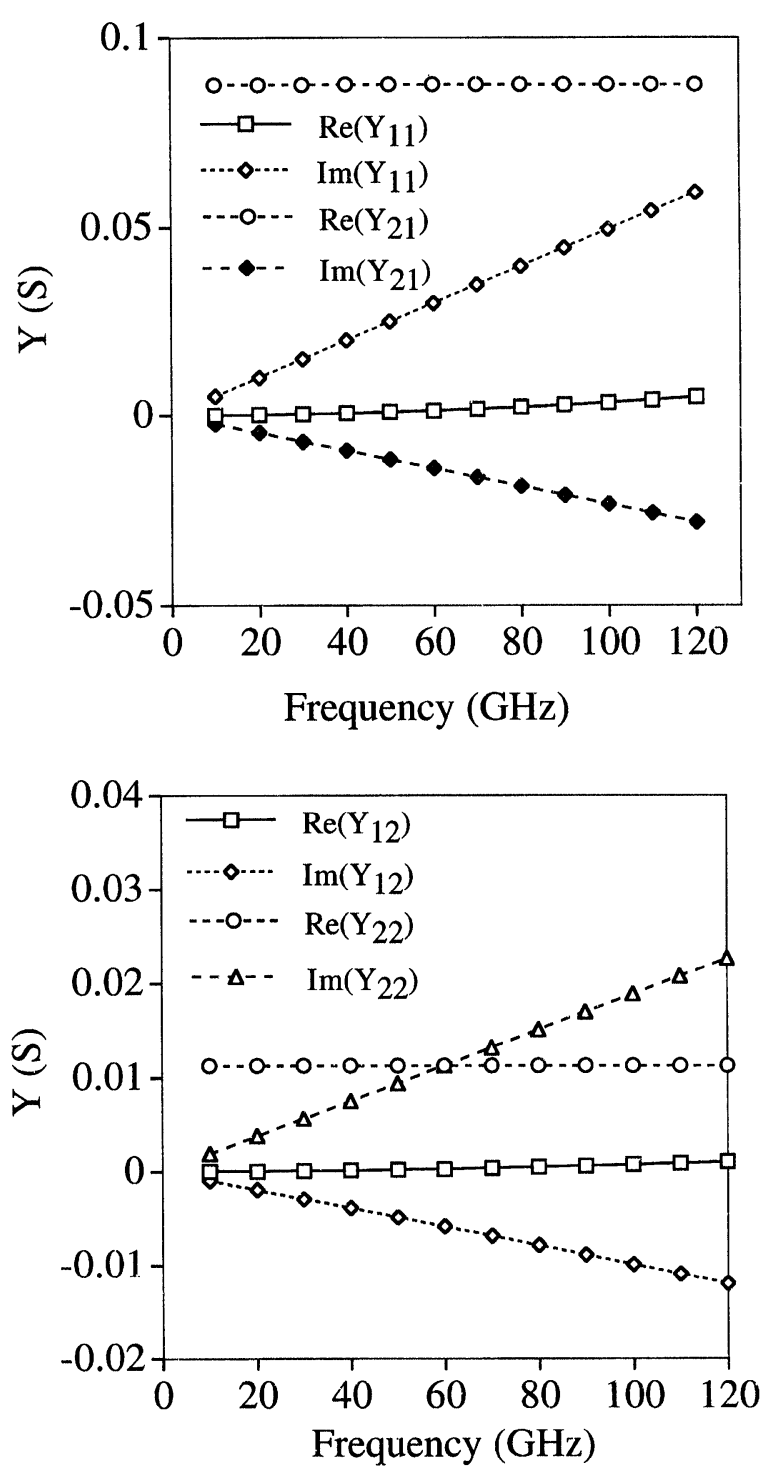

FIGURE $3 \quad Y$-parameters as a function of frequency extracted from the Fourier decomposition of the current transients at $V_{g}=-0.2$ and $V_{d}=1.5 \mathrm{~V}$. 
TABLE I Small signal equivalent circuit components calculated from the EMC simulations, $V_{g}=-0.2$ and $V_{d}=1.5 \mathrm{~V}$. Given also are the experimental values. $f T$ and $f_{\max }$ are given at $R_{g}=R_{c}=5 \Omega$

\begin{tabular}{lccccccccc}
\hline & $\mathrm{C}_{\mathrm{gs}}(\mathrm{fF})$ & $\mathrm{C}_{\mathrm{gd}}(\mathrm{fF})$ & $\mathrm{C}_{\mathrm{ds}}(\mathrm{fF})$ & $\mathrm{g}_{\mathrm{mo}}(\mathrm{ms})$ & $\mathrm{g}_{\mathrm{ds}}(\mathrm{fF})$ & $\mathrm{R}_{\mathrm{i}}(\Omega)$ & $\tau(\mathrm{pS})$ & $\mathrm{fT}(\mathrm{GHz})$ & $\mathrm{f}_{\max }(\mathrm{GHz})$ \\
\hline $\mathrm{MC}$ & 75.5 & 14.2 & 15.1 & 87.6 & 11.2 & 2.3 & 0.17 & 142 & 164 \\
Exp. & 77.8 & 8.73 & 14 & 67 & 8.65 & 3.75 & 0.32 & 114 & 150 \\
\hline
\end{tabular}

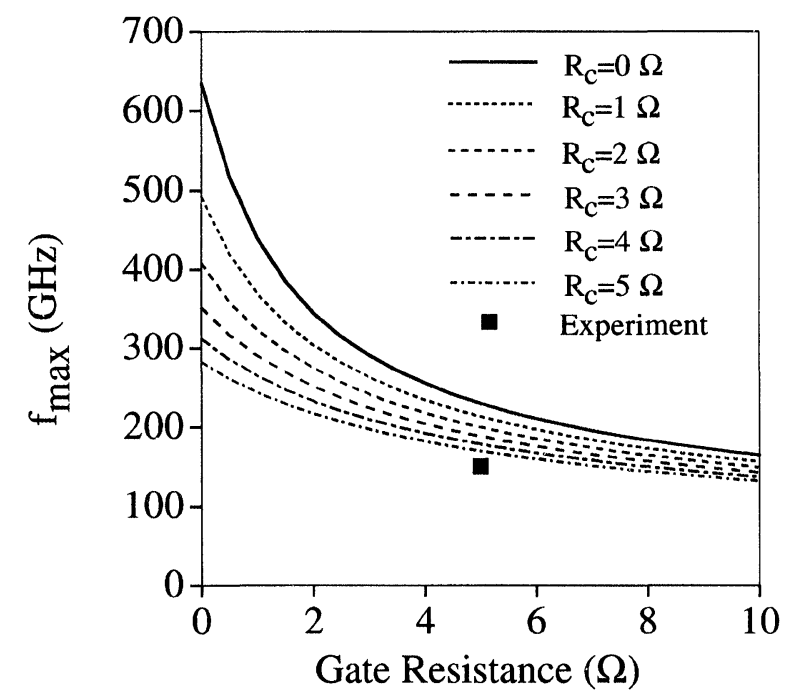

FIGURE 4 Effect of gate and contact resistances on $f_{\max }$.

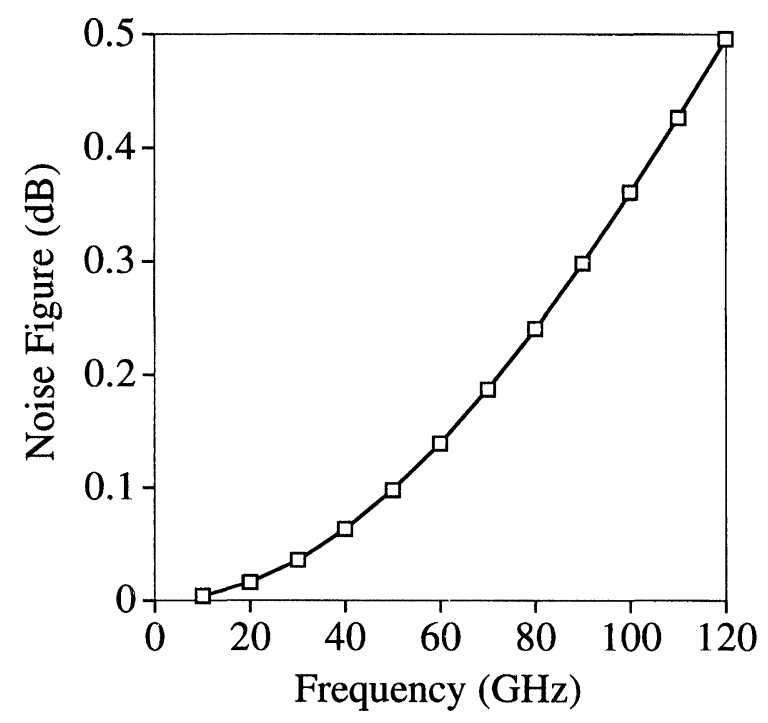

FIGURE 5 Intrinsic noise figure for the $120 \mathrm{~nm}$ pHEMT extracted from the current traces at $V_{g}=-0.2$ and $V_{d}=1.5 \mathrm{~V}$. equivalent circuit parameters extracted from the EMC simulations, $V_{g}=-0.2$ and $V_{d}=1.5 \mathrm{~V}$, together with the experimental values. The higher simulation $g_{\mathrm{mo}}$ and $f_{T}$ values is due to the fact that the MC simulations overestimate the velocity overshoot in the channel. The significant effect the gate and contact resistances have on the maximum frequency of oscillation is depicted in Figure 4. The intrinsic noise figure extracted from the transients is shown in Figure 5.

\section{CONCLUSION}

In this paper we have presented a comprehensive methodology for the RF analysis of FETs. The methodology is based on the transient EMC simulations of the intrinsic device followed by a post-processing stage during which the parasitic elements are included. It allows realistic estimation of the RF performance from MC simulations. The capabilities of the scheme are illustrated in example simulations for the $120 \mathrm{~nm}$ gate length pHEMT.

\section{References}

[1] Park, D. H. and Brennan, K. F. (1990). "Monte Carlo simulation of $0.35-\mu \mathrm{m}$ gate-length GaAs and InGaAs HEMT's", IEEE Trans. Electron Devices, 37, 618-628,

[2] Kizilyally, I. C., Artaki, M., Shah, N. J. and Chandra, A. (1991). "Scaling properties and short-channel effects in submicrometer AlGaAs/GaAs MODFET's: A Monte Carlo study", IEEE Trans. Electron Devices, 40, 234-249.

[3] Kim, K. W., Tian, H. and Litteljohn, M. A. (1991). "Analysis of delta doped and uniformly doped AlGaAs/ GaAs HEMT's by ensemble Monte Carlo simulation", IEEE Trans. Electron Devices, 38, 1737-1742.

[4] Babiker, S., Asenov, A., Barker, J. R. and Beaumont, S. P. (1996). "Finite element Monte Carlo simulation of recess gate compound FETs", Solid State Electronics, 39, 629635.

[5] Asenov, A., Reid, D., Barker, J. R., Cameron, N. and Beaumont, S. P. (1993). "Finite element simulation of 
recess gate MESFETs and HEMTs. The Simulator H2F", in Simulation of Semiconductor devices and processes, S. Selberherr, H. Stippel, E. Strasser, eds., Wien: Springer Verlag, 5, 265-268.

[6] Kim, H., Min, H., Tang, T. and Park, Y. (1992). "An Extended Proof of the Ramo-Shockley Theorem", Solid State Electronics, 34(11), 1251-1253.

[7] González, T. and Pardo, D. (1990). "Monte Carlo determination of the intrinsic small-signal equivalent circuit of MESFET's," IEEE Trans. Electron Devices, 42, 605611.

[8] Cameron, N., Taylor, M. R. S., Mclelland, H., Holland, M., Thayne, I., Elgaid, K. and Beaumont, S. P. (1995). "A high performance, high yield, dry etched pseudomorphic HEMT for W-band use", IEEE Trans. Microwave Theory and Techniques Symposium Digest, Orlando, FL, 435-438.

\section{Authors' Biographies}

Sharief Babiker has studied Electrical Engineering in Khartoum University Sudan (1979-1984). During the period 1985-90 he worked at the Electrical Engineering Department of Khartoum University. In 1994 he was awarded the Ph.D. degree for work on the theory and modelling of single-electronic devices and systems. His current research interests concentrate on the simulation and modelling of FETs with emphasis on submicron gate length InGaAs channel pHEMTs for RF applications.

Asen Asenov had 10 years industrial experience as a head of the Process and Device Modelling Group in IME-Sofia, developing one of the first integrated process and device CMOS simulators IMPEDANCE. He was visiting professor at the Physics Department of TU Munich, and is currently a Reader in the Department of Electronics and Electrical Engineering, Glasgow University. As a leader of the Device Modelling Group he has contributed to the development of $2 \mathrm{D}$ and $3 \mathrm{D}$ device simulators and their application in the design of FETs, SiGe MOSFETs and IGBTSs.

Nigel Cameron graduated from the University of Bath in 1984 and joined British Telecom's research laboratories to work on plasma process research and development for silicon fabrication. In March 1988 he moved to the Nanoelectronics Research Centre at the University of Glasgow where his responsibilities include the development of ultrafast transistors in compound semiconductors and their integration into manufacturable processes for millimetre-wave integrated circuits (MMICs). His interests extend across a broad range of semiconductor technologies encompassing materials and device physics; circuit design and test; manufacturing; and dc and high frequency characterisation. Nigel Cameron is the author or co-author of more than 35 technical papers.

Steven Beaumont was educated at the University of Cambridge and has been with the Department of Electronics and Electrical Engineering at the University of Glasgow since 1978. He became Head of Department in 1995 and he convenes the Nanoelectronics Research Centre's management committee. His research interests lie in the field of nanometre-scale fabrication and its application to electronic and optoelectronic devices. He has over 100 publications on electron beam nanolithography, dry etching, short-gate III-V based transistors, quantum transport devices, the optical properties of quantum dots, and single electron devices. Latterly he has become involved with the issue of manufacturability of mm-wave circuits and the use of nanometre-scale fabrication techniques coupled with technology-based device simulations to forecast performance and yield with the minimum of process iterations.

John Barker is Professor of Electronics in the Department of Electronics and Electrical Engineering. He has a long standing interest in computational methods, device modelling and transport theory. From $1970-85$ he was a member of the Theory group in the Dept. of Physics, University of Warwick, aside from 1978-79 when he worked at IBM T. J. Watson Laboratory, North Texas State University and Colorado State University. From 1987-89 he was academic director of the IBM UK/Glasgow University Kelvin Project on Numerically Intensive Parallel Computing. He is academic director of the Parallel Processing Centre at the University of Glasgow. 

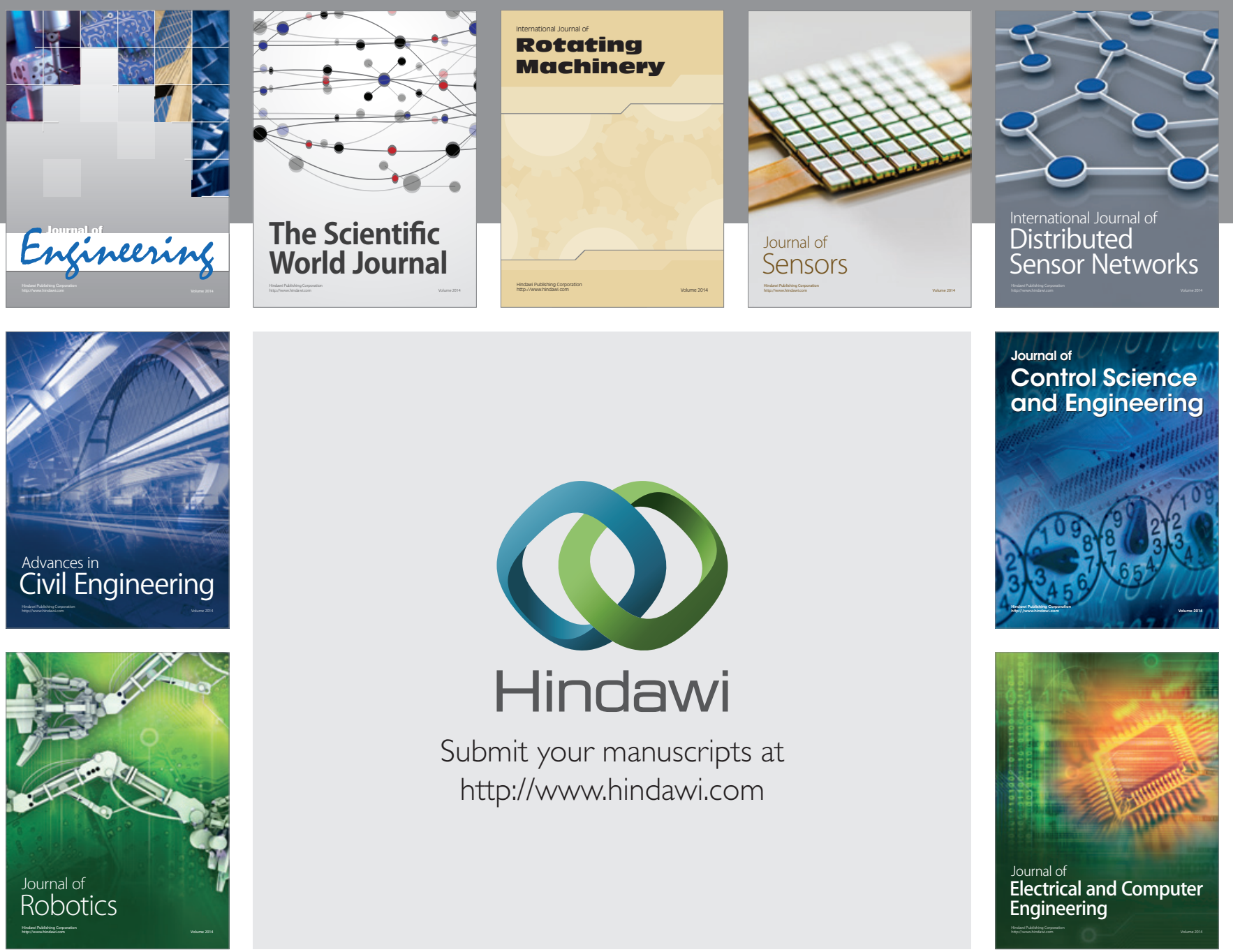

Submit your manuscripts at

http://www.hindawi.com
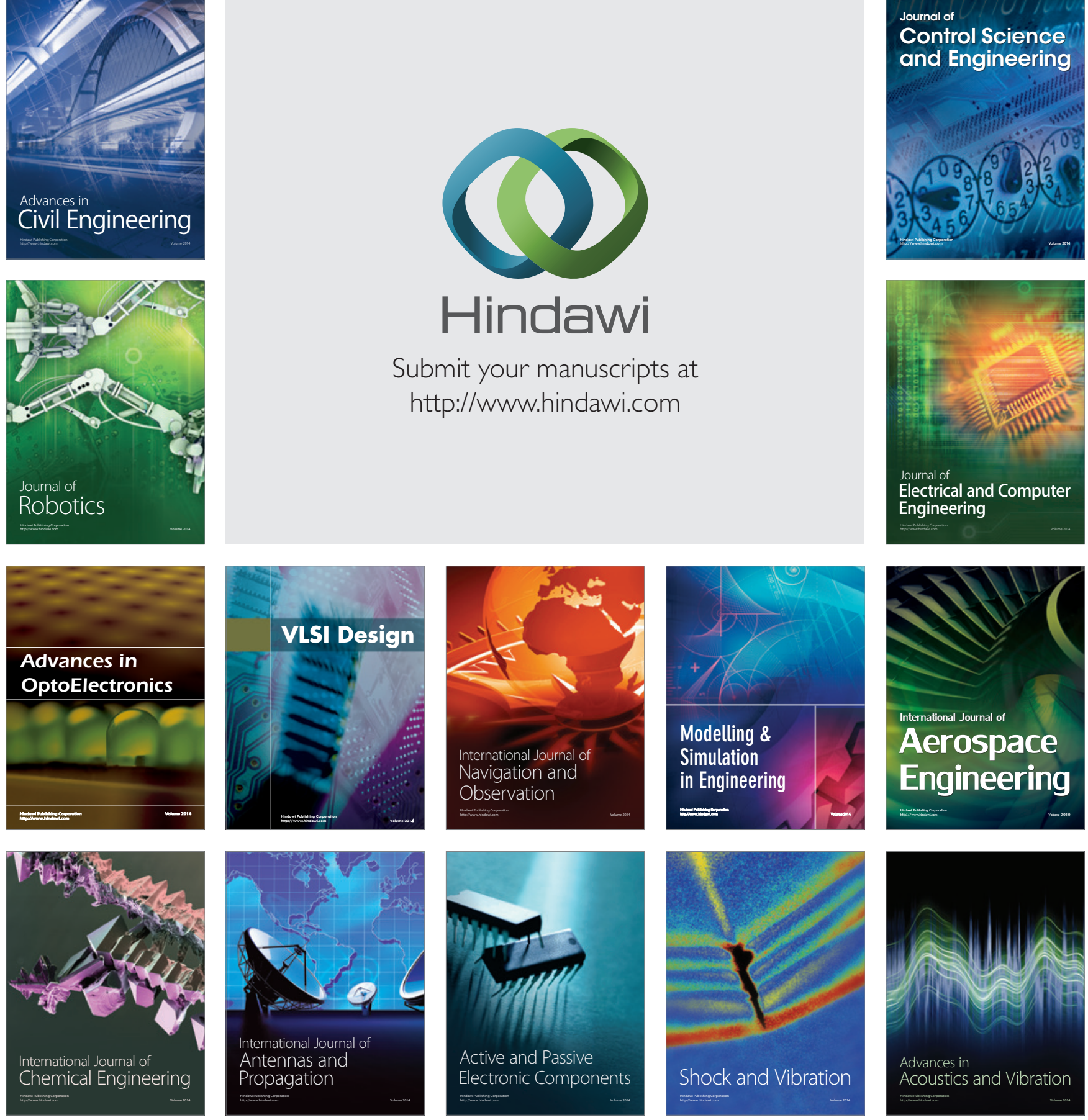